\title{
Correlações limnológicas em viveiros de cultivo do camarão-da-amazônia Macrobrachium amazonicum
}

\author{
Erlei Cassiano Keppeler \\ Universidade Federal do Acre, Centro Multidisciplinar \\ Colônia São Francisco, Gleba \\ CEP 69980-000, Campus de Cruzeiro do Sul - AC, Brasil \\ erleikeppeler@gmail.com
}

Submetido em 29/12/2007

Aceito para publicação em 23/07/2008

\section{Resumo}

A qualidade da água dos viveiros é fundamental para o sucesso da aqüicultura. Entre as variáveis as mais importantes estão o oxigênio dissolvido e o nitrogênio amoniacal. Neste trabalho, foi realizado um estudo limnológico de viveiros de cultivo de Macrobrachium amazonicum.. Doze viveiros com cerca de 0,01 ha foram povoados com 20 juvenis de M. amazonicum. $\mathrm{m}^{-2}$. Os animais foram alimentados com ração extrusada na proporção de 6 a 9\% da biomassa até a 14 ${ }^{\text {a }}$ semana. Depois de 145 dias de estocagem dos camarões, todos os viveiros foram drenados e despescados. Semanalmente, foram determinadas as seguintes variáveis da água: temperatura, oxigênio dissolvido, demanda bioquímica de oxigênio, $\mathrm{pH}$, alcalinidade total, condutividade elétrica, nitrato, nitrito, nitrogênio amoniacal, nitrogênio total, ortofosfato solúvel, fósforo total, clorofila a, clorofila b, clorofila c, feofitina, turbidez e sólidos totais suspensos. Este trabalho analisou, em viveiros de Macrobrachium amazonicum, a correlação entre diversas variáveis limnológicas. Semanalmente, os dados foram obtidos de doze viveiros com cerca de 0,01 ha e profundidade de $1,0 \mathrm{~m}$, povoados com 20 juvenis de $M$. amazonicum. m $^{-2}$, alimentados com ração extrusada, na taxa de 6 a $9 \%$ da biomassa até a 14ª semana. Depois de 145 dias de cultivo, os viveiros foram drenados e despescados. A ração diária teve pouco efeito sobre a qualidade da água dos viveiros do que da água de renovação. Das variáveis limnológicas analisadas, as que apresentaram as maiores correlações significativas foram entre o oxigênio dissolvido e nitrogênio amoniacal e entre o nitrito e a turbidez.

Unitermos: Macrobrachium amazonicum, variáveis limnológicas, correlações

\section{Abstract}

Correlation among water quality parameters of grow-out ponds of Macrobrachium amazonicum. Pond water quality is basic for the success of aquaculture, especially in ponds containing freshwater prawns. The variables considered most important are the dissolved oxygen and the ammonia-nitrogen. Twelve 0.01 ha earthen ponds were stocked with 20 juveniles.m ${ }^{-2}$. Prawns were fed on commercial diet at a rate of 7 to $9 \%$ of biomass until the $14^{\text {th }}$ week. After 145 days of stocking, all ponds were drained and harvested. The following water parameters were determined weekly: temperature, dissolved oxygen, oxygen biochemical demand, $\mathrm{pH}$, total alkalinity, electrical conductivity, nitrate-N, nitrite-N, ammonia-N, total nitrogen, soluble orthophosphate, total phosphate, chlorophyll a, chlorophyll b, chlorophyll c, pheophytin, total suspended solids and turbidity. Water exchange and daily feeding affected water quality slightly. Correlations among water quality parameters were either weak or absent. The limnological variables analyzed showed that the highest correlations were between the dissolved oxygen and ammonia-nitrogen and between nitrite and turbidity.

Key words: Macrobrachium amazonicum, water quality parameters, correlations 


\section{Introdução}

A qualidade da água dos viveiros é fundamental para o sucesso da aqüicultura (Boyd e Zimmermann, 2000; Kubitza, 2003). Entre as variáveis mais importantes estão o oxigênio dissolvido e o nitrogênio amoniacal. A importância do oxigênio reside no fato de a maioria dos animais satisfazer sua necessidade de energia por meio da oxidação de alimentos, com a formação de dióxido de carbono e água no processo (Schmidt-Nielsen, 2002). Portanto, este é de importância fundamental para a manutenção da vida (Margalef, 1983). O nitrogênio amoniacal é o principal resíduo nitrogenado excretado (Midlen e Redding, 2000) pelos camarões, tendo origem nos processos metabólicos de transformação e oxidação da proteína (aminoácidos) obtida nos alimentos. A ingestão de alimentos com excessivo teor protéico e/ou com desbalanço na sua composição em aminoácidos, geralmente, aumenta a excreção de nitrogênio amoniacal (Schmidt-Nielsen, 2002) pelos camarões. Outra importante fonte de nitrogênio amoniacal no cultivo é a decomposição microbiana das proteínas e aminoácidos eliminados nas fezes (Hargreaves, 1998). O nitrogênio amoniacal é tóxico aos camarões, o que obriga a adoção de estratégias que evitem o seu acúmulo excessivo na água ao longo do cultivo (Kubitza, 2003).

Além dos compostos nitrogenados, outras características limnológicas estão envolvidas na dinâmica de viveiros para permitir o desenvolvimento dos camarões (Boyd e Zimmermann, 2000). A turbidez pode ser influenciada pelo material alóctone (Boyd, 1979) proveniente da água de abastecimento ou da ração. Sua variação, quando advém da água que mantém os viveiros, pode ter origem na erosão das margens da represa, causada pela ação do vento e das chuvas, bem como o transporte dos sedimentos (Ono e Kubitza, 2003).

O fósforo é geralmente o principal fator limitante ao desenvolvimento do fitoplâncton em ambientes aquáticos (Esteves, 1998). Entre todas as formas ou frações de fosfato, o ortofosfato assume maior relevância por ser a principal forma assimilada pelos vegetais aquáticos (Wetzel, 1981). Sua concentração depende da densidade e da atividade de organismos, especialmente fitoplanctônicos e de macrófitas aquáticas, os quais durante a fotossíntese podem assimilar grandes quantidades destes íons (Boyd, 1979; Esteves, 1998). Em ambientes aquáticos de alta temperatura, a taxa metabólica dos organismos aumenta consideravelmente, fazendo com que o ortofosfato solúvel seja mais rapidamente assimilado e incorporado na sua biomassa (Boyd, 1979). Este é um dos principais motivos pelo qual a concentração de ortofosfato solúvel é muito baixa.

Adicionalmente, Zimmermann (1998) e Boyd e Zimmermann (2000) listam variáveis também fundamentais para o sucesso do cultivo de Macrobrachium, que são o oxigênio, $\mathrm{pH}$, alcalinidade total, sólidos totais suspensos. Em suma, aspectos biológicos, químicos e físicos da água influenciam no crescimento, na resistência a doenças, na reprodução e na tolerância a variação da temperatura dos camarões de água doce extremas (Boyd e Zimmerman, 2000).

O objetivo do presente trabalho foi estudar a correlação entre diversas variáveis limnológicas nos viveiros de $M$. amazonicum. Estudos desta natureza também foram realizados por Enrich-Prast et al. (2004) quando pesquisaram lagoas costeiras da restinga de Jurubatiba, na correlação de variáveis limnológicas, utilizando também como ferramenta estatística o coeficiente de correlação de Pearson (r).

\section{Material e Métodos}

\section{Descrição dos cultivos}

O experimento foi conduzido no Setor de Carcinicultura do Centro de Aqüicultura da UNESP (CAUNESP) no período de dezembro/2003 a maio/2004. Foram utilizados doze viveiros de aproximadamente 0,01 ha, escavados, com fundo de terra, sem revestimento nas laterais, e profundidade com cerca de $1,0 \mathrm{~m}$. Estes foram construídos sobre latossedimento vermelhoescuro com textura argilosa. As características gerais do sedimento foram descritas em Valenti (1989) e apresentadas na Tabela 1. Os taludes foram protegidos com grama para prevenção de erosão. 
TABELA 1: Análise do sedimento (química e granulométrica) formador do fundo dos viveiros (Valenti, 1989).

\begin{tabular}{c|c|c} 
Parâmetro & Unidade & $\begin{array}{c}\text { Valor } \\
\text { determinado }\end{array}$ \\
\hline Potássio & Meq. $100 \mathrm{~cm}^{-3}$ & 0,3 \\
\hline Cálcio & Meq. $100 \mathrm{~cm}^{-3}$ & 2,6 \\
\hline Magnésio & Meq. $100 \mathrm{~cm}^{-3}$ & 0,7 \\
\hline Hidrogênio + Alumínio & Meq. $100 \mathrm{~cm}^{-3}$ & 1,9 \\
\hline Saturação & $\%$ & 65 \\
\hline pH (em $\left.\mathrm{CaCl}_{2}\right)$ & - & 5,5 \\
\hline Argila & $\%$ & 58,10 \\
\hline Silte (limpo) & $\%$ & 30,26 \\
\hline Areia & $\%$ & 11,64 \\
\hline
\end{tabular}

Os viveiros localizam-se na cidade de Jaboticabal (21 ${ }^{\circ} 15^{\prime} 22^{\prime}$ 'S e 48 $18^{\prime} 48^{\prime}$ 'W), Estado de São Paulo, no Campus da Universidade Estadual Paulista (Figura 1). O clima é do tipo Cwa, segundo a classificação de Koepen, existindo duas estações, seca e úmida, que se alternam. A precipitação e temperaturas atmosféricas médias anuais são de $1350 \mathrm{~mm}$ e $21 / 22^{\circ} \mathrm{C}$, respectivamente. A variação das médias das temperaturas do ar máximas em janeiro é de 29 a $30^{\circ} \mathrm{C}$ e a média das mínimas em julho é de 11 a $12^{\circ} \mathrm{C}$ (Troppmair, 1975).

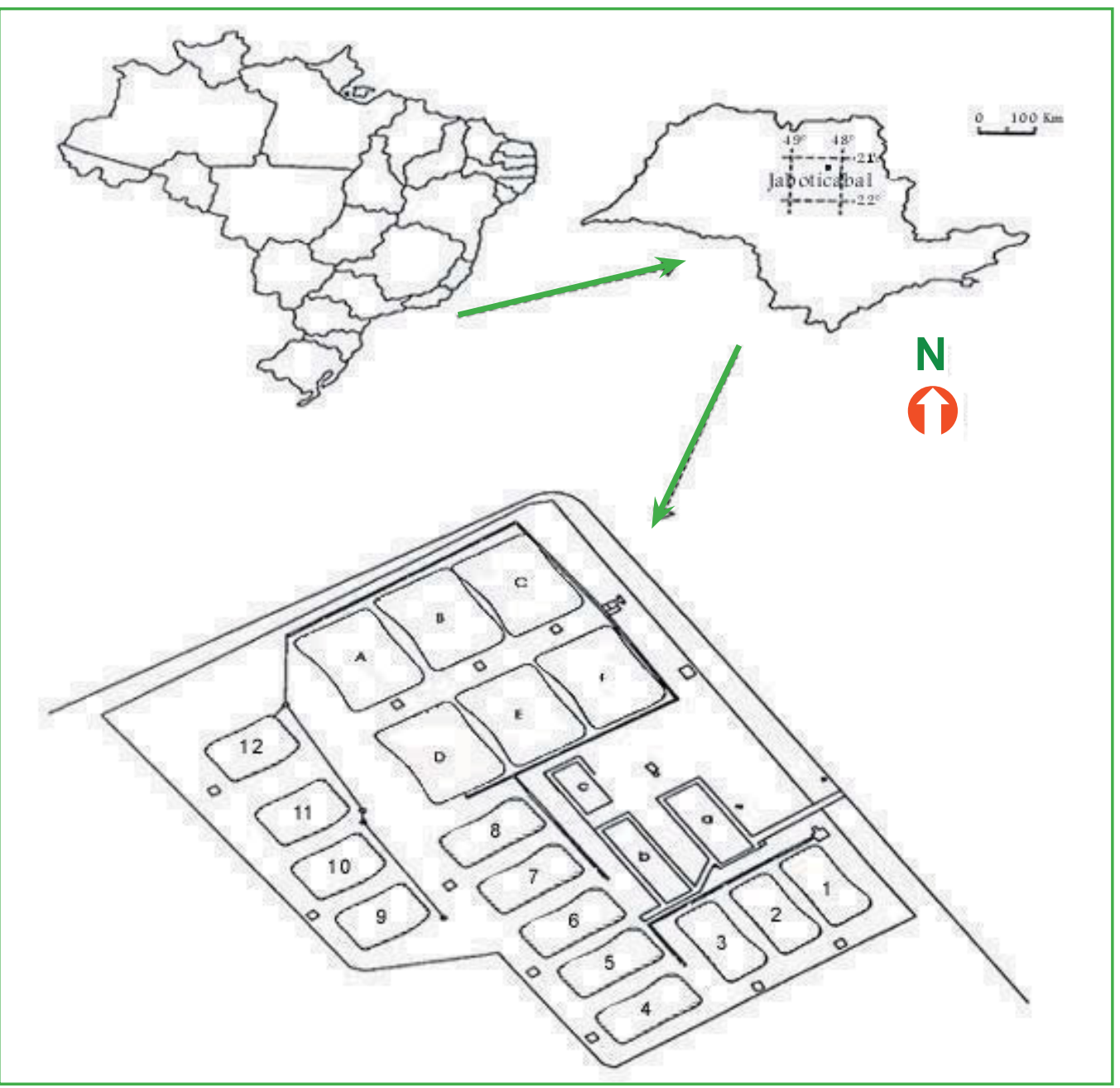

FIGURA 1: Localização da área de estudo (Legenda: 1 a 12 Viveiros de crescimento final do M. amazonicum; A a F - Viveiros usados para reprodução de $M$. amazonicum, M. rosenbergii e esporadicamente usado como berçário secundário; A - Laboratório de larvicultura e qualidade de água; B - Berçário; C - Laboratório de Biometria. 
Seguiu-se rigorosamente o manejo usado no sistema semi-intensivo de camarões-de-água doce (New, 2002). Antes do enchimento, os viveiros foram drenados, secos ao ar, e o excesso de sedimento foi retirado. Em seguida, realizou-se a calagem através da aplicação de 1,0ton/ha de calcário dolomítico e fertilização orgânica com adição de 3,0ton/ha de esterco bovino curtido.

Os viveiros foram abastecidos com água proveniente de duas represas que reciclam os efluentes de outros viveiros e contêm peixes. Estas apresentam características hipertróficas, e sua limnologia está descrita em Macedo (2004). A taxa de renovação foi $5-10 \%$ do volume total por dia. Portanto, o tempo de retenção foi de 10 a 20 dias. Esporadicamente, quando algum viveiro apresentou oxigênio dissolvido abaixo de $2,0 \mathrm{mg} . \mathrm{L}^{-1}$, pela manhã, acionou-se um aerador de emergência.

Os viveiros foram povoados com 20 juvenis de M. amazonicum. $\mathrm{m}^{-2}$, com 50 dias após a metamorfose em berçários primários e secundários, com peso médio de $0,36 \pm 0,09 \mathrm{~g}$. Os animais foram alimentados com ração extrusada com $37 \% \mathrm{~PB}$, cuja composição é apresentada na Tabela 2. O arraçoamento foi a lanço, duas vezes ao dia. Os viveiros receberam adubação química para incentivar o crescimento de organismos bentônicos (larvas de insetos, anelídeos, etc.) que servem de alimento natural para o camarão. Esta adubação foi realizada com uréia + sulfato de amônia, NPK e uréia + superfosfato simples. As quantidades de $\mathrm{Ne} \mathrm{P}$ aplicadas semanalmente são apresentadas na Tabela 3.

TABELA 2: Composição da ração Laguna da Socil CRS-38 usada neste experimento.

\begin{tabular}{c|c}
\hline \multicolumn{2}{c}{ Níveis de Garantia da ração Comercial Laguna da } \\
Socil (\%) & 13 \\
\hline Umidade (máxima) & 37 \\
\hline Proteína bruta (mínimo) & 7 \\
\hline Extrato etéreo (mínimo) & 7 \\
\hline Fibra bruta (máximo) & 14 \\
\hline Matéria mineral (máxima) & 4 \\
\hline Cálcio (máximo) & 1 \\
\hline Fósforo (mínimo) &
\end{tabular}

TABELA 3: Quantidade de nitrogênio $(\mathrm{N})$ e fósforo $(\mathrm{P})$ das fertilizações semanais.

\begin{tabular}{c|c|c}
\hline Semanas & $\mathbf{N}\left(\mathbf{k g . h a ^ { - 1 }}\right)$ & $\mathbf{P}\left(\mathbf{k g . h a ^ { - 1 }}\right)$ \\
\hline 3 & 8,8 & - \\
\hline 4 & 8,8 & - \\
\hline 5 & 3,12 & 0,68 \\
\hline 6 & 3,12 & 0,68 \\
\hline 9 & 3,12 & 0,68 \\
\hline 11 & 1,98 & 4,2 \\
\hline 12 & 1,98 & 4,2 \\
\hline 14 & 1,98 & 4,2 \\
\hline
\end{tabular}

Fonte: Keppeler (2008)

Após a $14^{\mathrm{a}}$ semana os viveiros foram submetidos a despesca seletiva e mista e arraçoamento a lanço reportados nos trabalhos de Keppeler (2005) e Keppeler e Valenti (2006). Quando parte da população atingiu o tamanho comercial, em meados de março, foram iniciadas as despescas seletivas.

\section{Coleta de dados}

Semanalmente, foram realizadas medições e coletadas amostras da água de abastecimento, do interior dos viveiros e dos efluentes nos períodos da manhã (7h-9h) e da tarde (15-17h). As variáveis limnológicas determinadas e os métodos usados são apresentados a seguir: temperatura (superfície e fundo), o teor de oxigênio dissolvido (fundo) e demanda bioquímica de oxigênio $\left(\mathrm{DBO}_{5}\right)$ foram mensurados com oxímetro marca YSI modelo 52 (amostra integrada). Para o cálculo da $\mathrm{DBO}_{5}$, considerou-se a diferença inicial e final do oxigênio dissolvido no período de cinco dias de incubação, a uma temperatura de $20 \pm 1^{\circ} \mathrm{C}$, com água de diluição geralmente a 40\% (APHA, 1992). O pH e a condutividade elétrica foram aferidos no fundo dos viveiros, com peagômetro e condutivímetro marca YSI, modelo 63.

Para análise das formas de nitrogênio e fósforo foram coletadas amostras integradas, incluindo a coluna d'água e água do fundo. Os métodos utilizados nas análises encontram-se descritos em APHA (1998) para nitrato com emprego do sulfato de hidrazina, em Strickland e Parsons (1960) para nitrito, em Solorzano (1969) para nitrogênio amoniacal e, em 
Valderrama (1981) para nitrogênio total. O fósforo total e ortofosfato solúvel foram determinados segundo Adams (1990) e Boyd e Tucker (1992); a turbidez foi avaliada segundo Wetzel e Likens (1991) por meio do espectrofotômetro, marca Hach, modelo 2000, e os sólidos totais suspensos, pelo método gravimétrico, segundo Boyd e Tucker (1992). As clorofilas $a, b, c$ e a feofitina foram analisadas de acordo com APHA (1992). Todas as determinações foram realizadas em duplicatas. Quando os valores obtidos foram próximos, considerou-se como resultado o valor médio. Se os valores fossem discrepantes, realizou-se uma terceira leitura e descartou-se o valor extremo.

\section{Funcionamento dos viveiros}

Para estudar o funcionamento dos viveiros, utilizaram-se os dados dos nove viveiros, operados com despesca total, ao final de 145 dias, nas primeiras 14 semanas, isto é, antes do início do arraçoamento diferencial. Assim, o estudo foi realizado com nove réplicas, sendo que para cada uma representa-se o sistema de cultivo semi-intensivo do camarão-daamazônia.

\section{Análise estatística}

Todos os dados foram analisados pelo coeficiente de correlação linear de Pearson (r) com alfa de $0,05 \%$, utilizando-se os "softwares" "Statistica" versão 6.0 da Statsoft Company (Statsoft, 1996). Foram considerados resultados estatisticamente significativos aqueles para os quais o valor da probabilidade (p) foi igual ou menor que 0,05 (Zar, 1984).

\section{Resultados}

A quantidade fornecida da ração, dos nove viveiros, até a décima quarta semana de cultivo atingiu $80 \mathrm{~kg}$. ha $^{-1} \cdot$ dia $^{-1}$, mas a média, considerando todo o período do cultivo foi de $29 \mathrm{~kg} \cdot \mathrm{ha}^{-1}$.dia ${ }^{-1}$ (cerca de $290 \mathrm{~g}$ por viveiro). A quantidade total de ração, adicionada em cada viveiro, até a $14^{\mathrm{a}}$ semana foi, em média, $39,69 \mathrm{~kg}$.

A Tabela 4 mostra as médias das variáveis limnológicas, no período da manhã e tarde nos nove viveiros, até a $14^{\mathrm{a}}$ semana. Os desvios padrão foram altos, indicando grau de variabilidade em todas as variáveis.

A Tabela 5 apresenta as correlações entre a ração e algumas variáveis limnológicas nos viveiros. A correlação significativa $(p<0,05)$ foi apenas para o oxigênio dissolvido e nitrogênio amoniacal.

A Tabela 6 apresenta os coeficientes de correlação linear de Pearson (r), obtidos entre as variáveis limnológicas. O oxigênio dissolvido teve correlação negativa com nitrogênio amoniacal $(-0,29)$ e com o ortofosfato solúvel $(-0,35$ a $-0,38)$. O nitrogênio amoniacal também apresentou correlação positiva com o nitrito $(0,34)$ e turbidez $(0,34)$. O nitrito teve uma alta correlação com a turbidez $(1,0)$. O nitrato apresentou correlação positiva com a DBO $(0,40)$ e com os sólidos totais suspensos $(0,39)$. A feofitina apresentou correlação significativa e positiva com a clorofila a $(0,99)$, clorofila b $(0,33)$ e clorofila c $(0,31)$.

TABELA 4: Médias ( \pm desvios padrão) dos valores obtidos das variáveis limnológicas nos nove viveiros pela manhã e tarde, até a $14^{\mathrm{a}}$ semana.

\begin{tabular}{l|c|c}
\hline & Manhã & Tarde \\
\hline Oxigênio dissolvido $\left(\mathrm{mg} . \mathrm{L}^{-1}\right)$ & $7,83 \pm 2,54$ & $9,04 \pm 2,40$ \\
\hline Oxigênio dissolvido $(\%)$ & $95,80 \pm 32,64$ & $117,94 \pm 34,32$ \\
\hline $\mathrm{DBO}_{5}\left(\mathrm{mg} . \mathrm{L}^{-1}\right)$ & $6,47 \pm 3,80$ & $7,53 \pm 4,29$ \\
\hline $\mathrm{pH}$ & $7,30 \pm 0,67$ & $7,55 \pm 0,50$ \\
\hline Alcalinidade total $\left(\mathrm{mg} \cdot \mathrm{L}^{-1} \mathrm{CaCO}_{3}\right)$ & $48,35 \pm 7,97$ & $48,76 \pm 10,01$ \\
\hline Condutividade elétrica $\left(\mu \mathrm{S} \cdot \mathrm{cm}^{-1}\right)$ & $98,76 \pm 19,31$ & $102,70 \pm 19,77$ \\
\hline Nitrato $\left(\mu \mathrm{g} \cdot \mathrm{L}^{-1}\right)$ & $616 \pm 1012$ & $947 \pm 1233$ \\
\hline Nitrito $\left(\mu \mathrm{g} . \mathrm{L}^{-1}\right)$ & $40 \pm 21$ & $42 \pm 27$ \\
\hline Nitrogênio amoniacal $\left(\mu \mathrm{g} . \mathrm{L}^{-1}\right)$ & $130,30 \pm 112,31$ & $80,89 \pm 80,66$ \\
\hline Ortofosfato solúvel $\left(\mathrm{mg} . \mathrm{L}^{-1}\right)$ & $0,040 \pm 0,039$ & $0,031 \pm 0,034$ \\
\hline Fósforo total $\left(\mathrm{mg} . \mathrm{L}^{-1}\right)$ & $0,158 \pm 0,152$ & $0,121 \pm 0,126$ \\
\hline Clorofila a $\left(\mu \mathrm{g} . \mathrm{L}^{-1}\right)$ & $231 \pm 336$ & $210 \pm 257$ \\
\hline Clorofila b $\left(\mu \mathrm{g} . \mathrm{L}^{-1}\right)$ & $114 \pm 204$ & $89 \pm 96$ \\
\hline Clorofila c $\left(\mu \mathrm{g} . \mathrm{L}^{-1}\right)$ & $70 \pm 96$ & $53 \pm 59$ \\
\hline Feofitina $\left(\mu \mathrm{g} \cdot \mathrm{L}^{-1}\right)$ & $585 \pm 789$ & $624 \pm 707$ \\
\hline Sólidos totais suspensos $\left(\mathrm{mg} . \mathrm{L}^{-1}\right)$ & $0,025 \pm 0,049$ & $0,017 \pm 0,038$ \\
\hline Turbidez $(\mathrm{UNT})$ & $22 \pm 17$ & $18 \pm 10$ \\
\hline
\end{tabular}


TABELA 5: Coeficientes de correlação linearde Pearson (r) obtidos para expressar as correlações entre a quantidade de ração fornecida diariamente e parâmetros limnológicos, nos viveiros.

\begin{tabular}{l|c}
\multicolumn{1}{c|}{ Variáveis } & R \\
\hline Oxigênio dissolvido & $-0,14^{*}$ \\
\hline Nitrogênio amoniacal & $0,35^{*}$ \\
\hline Nitrato & $-0,09$ \\
\hline Fósforo total & $-0,08$ \\
\hline Clorofila a & $-0,05$ \\
\hline Feofitina & 0,10 \\
\hline Sólidos totais suspensos & 0,10 \\
\hline
\end{tabular}

*Indica que "r" é significativo ao nível de 5\% de significância.
A Tabela 7 mostra a correlação entre a quantidade de ração e algumas variáveis limnológicas nos efluentes. A ração apresentou correlação negativa significativa com o oxigênio dissolvido e, positiva com o nitrogênio amoniacal.

No final do cultivo foram obtidas as produtividades expressas na Tabela 8 .

\section{Discussão}

Correlações altas entre a feofitina e clorofila $a$ foram observadas. A feofitina resulta da degradação da clorofila a (Wetzel, 1981) e, portanto, aumentando-se o substrato deve-se aumentar o produto.

TABELA 6: Coeficientes de correlação linear de Pearson (r) obtidos para expressar as correlações entre as variáveis limnológicas estudadas entre si no interior dos viveiros no período da tarde.

\begin{tabular}{|c|c|c|c|c|c|c|c|c|c|c|c|c|c|c|c|c|c|}
\hline & OD & $\mathrm{OD} \%$ & DBO5 & $\mathrm{pH}$ & Alcal & Amon & Nitrat & Nitrito & Ortofos & Fosf & Clora & Clorb & Clorc & Feof & Std & Turb & \\
\hline OD & 1,00 & & & & & & & & & & & & & & & & OD \\
\hline $\mathrm{OD} \%$ & $0,99 *$ & 1,00 & & & & & & & & & & & & & & & $\mathrm{OD} \%$ \\
\hline DBO5 & 0,04 & 0,06 & 1,00 & & & & & & & & & & & & & & DBO5 \\
\hline $\mathrm{pH}$ & $-0,08$ & $-0,07$ & 0,04 & 1,00 & & & & & & & & & & & & & $\mathrm{pH}$ \\
\hline Alcal & 0,20 & 0,23 & 0,24 & $-0,11$ & 1,00 & & & & & & & & & & & & Alcal \\
\hline Amon & $-0,25$ & $-0,29 *$ & $-0,09$ & $-0,20$ & $-0,09$ & 1,00 & & & & & & & & & & & Amon \\
\hline Nitra & 0,02 & 0,05 & $0,40 *$ & $-0,14$ & 0,24 & $-0,25$ & 1,00 & & & & & & & & & & Nitrat \\
\hline Nitri & $-0,05$ & $-0,05$ & $-0,14$ & $-0,11$ & $-0,03$ & $0,34^{*}$ & $-0,10$ & 1,00 & & & & & & & & & Nitrit \\
\hline Ortofosf & $-0,35^{*}$ & $-0,38 *$ & $-0,02$ & $-0,01$ & $-0,16$ & 0,15 & $-0,10$ & 0,08 & 1,00 & & & & & & & & Ortofosf \\
\hline Fosf & 0,03 & 0,06 & 0,04 & 0,07 & 0,21 & 0,08 & $-0,05$ & $-0,03$ & $-0,14$ & 1,00 & & & & & & & Fosf \\
\hline Clora & 0,02 & 0,02 & $-0,10$ & $-0,06$ & $-0,13$ & $-0,07$ & $-0,02$ & $-0,01$ & $-0,05$ & $-0,12$ & 1,00 & & & & & & Clora \\
\hline Clorb & $-0,05$ & $-0,06$ & $-0,27$ & $-0,25$ & 0,21 & 0,22 & $-0,06$ & 0,10 & 0,07 & $-0,16$ & $0,31 *$ & 1,00 & & & & & Clorb \\
\hline Clore & 0,18 & 0,19 & 0,27 & $-0,09$ & $-0,20$ & $-0,12$ & 0,07 & 0,12 & $-0,01$ & $-0,22$ & 0,24 & 0,18 & 1,00 & & & & Clorc \\
\hline Feof & 0,01 & 0,01 & $-0,09$ & $-0,07$ & $-0,14$ & $-0,07$ & $-0,03$ & $-0,02$ & $-0,02$ & $-0,14$ & $0,99 *$ & 0,33 * & $0,31^{*}$ & 1,00 & & & Feof \\
\hline STD & $-0,20$ & $-0,20$ & 0,18 & $-0,08$ & 0,07 & $-0,08$ & $0,39 *$ & 0,17 & 0,02 & $-0,14$ & $-0,10$ & 0,02 & $-0,08$ & $-0,10$ & 1,00 & & Std \\
\hline Turb & $-0,05$ & $-0,05$ & $-0,14$ & $-0,11$ & $-0,03$ & $0,34^{*}$ & $-0,10$ & $1,00^{*}$ & 0,08 & $-0,03$ & $-0,01$ & 0,10 & 0,12 & $-0,02$ & 0,17 & 1,00 & Turb \\
\hline
\end{tabular}

$\mathrm{OD}=$ Oxigênio dissolvido em mg. $\mathrm{L}^{-1}$. Oxigênio dissolvido (\%); $\mathrm{DBO}_{5}=$ Demanda bioquímica de oxigênio; Alcal = Alcalinidade total; Amon $=$ Nitrogênio amoniacal; Nitra $=$ Nitrato; Nitri $=$ nitrito; Ortofosf $=$ ortofosfato solúvel; Fosf $=$ Fósforo total; Clora $=$ Clorofila a; Clorb $=$ Clorofila b; Clorc $=$ Clorofila c; Feof $=$ feofitina; $\mathrm{STD}=$ sólidos totais suspensos e turb = turbidez. *indica que " $\mathrm{r}$ " é significativo ao nível de 5\% de significância. 
TABELA 7: Correlação entre a ração e algumas variáveis limnológicas nos efluentes.

\begin{tabular}{c|c}
\hline Variáveis & r \\
\hline Oxigênio dissolvido & $-0,21^{*}$ \\
\hline N-amoniacal & $0,27^{*}$ \\
\hline Nitrato & 0,02 \\
\hline Fósforo total & $-0,02$ \\
\hline Clorofila a & 0,04 \\
\hline Feofitina & $-0,04$ \\
\hline Sólidos totais suspensos & 0,03 \\
\hline
\end{tabular}

*Indica que "r" é significativo ao nível de 5\% de significância.

TABELA 8: Dimensão, número de indivíduos estocados, produção para cada viveiro.

\begin{tabular}{c|c|c|c}
\hline Viveiros & $\begin{array}{c}\text { Dimensão do } \\
\text { viveiro } \mathbf{( m}^{\mathbf{2}} \mathbf{)}\end{array}$ & $\begin{array}{c}\mathbf{N}^{\mathbf{i}} \text { indivíduos } \\
\text { estocados }\end{array}$ & $\begin{array}{c}\text { Produção } \\
\mathbf{( k g . h a}^{-1} \mathbf{)}\end{array}$ \\
\hline 1 & 89 & 1780 & 717,93 \\
\hline 2 & 89 & 1780 & 793,05 \\
\hline 3 & 92 & 1840 & 637,03 \\
\hline 4 & 100 & 2000 & 642,53 \\
\hline 5 & 101 & 2020 & 729,13 \\
\hline 6 & 102 & 2040 & 612,6 \\
\hline 7 & 106 & 2120 & 695,53 \\
\hline 8 & 100 & 2000 & 666,27 \\
\hline 9 & 124 & 2480 & 597,11 \\
\hline 10 & 115 & 2300 & 615,76 \\
\hline 11 & 120 & 2400 & 500,70 \\
\hline 12 & 98 & 1960 & 630,29 \\
\hline
\end{tabular}

Fonte: Keppeler (2008) modificado

$\mathrm{O}$ arraçoamento promove excesso de produtos nitrogenados decorrentes da excreção, assim como da decomposição microbiana da matéria orgânica (Hargreaves, 1998). Os principais compostos tóxicos são nitrogênio amoniacal e nitrito (Shishehchian et al., 1999). Este último é um composto intermediário da oxidação da amônia a nitrato (Midlen e Redding, 2000), sendo observado em águas muito eutrofizadas ou nas camadas de água mais profundas, onde a decomposição anaeróbica leva ao seu acúmulo (Ono e Kubitza, 2003). $\mathrm{O}$ nitrogênio amoniacal também é um produto dessa decomposição, bem como da excreção nitrogenada (Boyd e Zimmermann, 2000). Embora nitrogênio amoniacal, nitrito e nitrato apresentem teoricamente correlações, neste estudo apenas o nitrogênio amoniacal e o nitrito apresentaram correlação significativa. No estudo, em lagoas costeiras da Restinga de Jurubatiba, efetuado por Enrich-Prast et al. (2004), também foi observada correlação entre oxigênio e nitrogênio amoniacal.

Por outro lado, a turbidez apresentou correlações significativas com o nitrito e o nitrogênio amoniacal. É possível que o horário do arraçoamento pela manhã proporcionou a presença da ração ocasionando turbidez e, conseqüente degradação da ração na coluna d'água. Apesar de neste estudo, não haver correlação entre $\mathrm{pH}$ e condutividade esta foi observada no estudo de Enrich-Prast et al. (2004), em pesquisas de lagoas costeiras.

A ração teve efeito muito pequeno sobre as variáveis limnológicas e do sedimento. A ração apresentou correlação significativa fraca apenas com o oxigênio dissolvido e nitrogênio amoniacal. Isto, possivelmente, ocorreu como conseqüência da decomposição dos restos não consumidos e fezes geradas, além da sua metabolização pelos camarões, que consomem oxigênio para oxidar o alimento ingerido e excretam nitrogênio amoniacal. Investigações sobre o consumo de oxigênio e compostos nitrogenados resultantes no metabolismo digestivo dos camarões foram reportados por Mallasen e Valenti (2006) e Araújo e Valenti (2007).

A produtividade dos camarões apresentou uma média de $653,16 \mathrm{~kg} \cdot \mathrm{ha}^{-1}$. Preto et al. (2008) também usaram arraçoamento a lanço no cultivo do camarãoda-amazônia e encontraram produtividade média de $586 \pm 42 \mathrm{~kg} \cdot \mathrm{ha}^{-1}$.

A partir dos resultados, foi possível concluir que:

1) A ração fornecida diariamente teve efeito muito pequeno sobre a qualidade de água, reduzindo levemente o oxigênio dissolvido. Por conseguinte, aumentou-se o teor de nitrogênio amoniacal.

2) A correlação entre as diversas variáveis na coluna d'água foi fraca, ou inexistente, exceto entre a clorofila a e a feotitina.

\section{Agradecimentos}

Ao Centro de Aqüicultura da UNESP pela infraestrutura oferecida para a realização deste trabalho. 


\section{Referências}

Adams, V. D. 1990. Water and wastewater examination manual. Lewis Publishers, Chelsea, USA, 247pp.

APHA. 1992. Standard methods for the examination of water and wastewater. $18^{\text {th }}$ ed. American Public Health Association, Washington, USA, 1219pp.

APHA. 1998. Standard methods for the examination of water and wastewater. $20^{\text {th }}$ ed. American Public Health Association, Washington, USA, 824pp.

Araújo, M. C.; Valenti, W. C. 2007. Feeding habitat of the Amazon River prawn Macrobrachium amazonicum larvae. Aquaculture, 38: 516-526.

Boyd, C. E. 1979. Water quality in warmwater fishponds. Auburn University, Alabama, USA, 359pp.

Boyd, C. E.; Tucker, C. S. 1992. Water quality and pond soil analyses for aquaculture. Auburn University, Opelika, USA, 183pp.

Boyd, C. E.; Zimmermann, S. 2000. Grow-out systems: Water quality and soil management. In: New, M. B. \& Valenti, W. C. (Eds). Freshwater prawn culture: The farming of Macrobrachium rosenbergii. Blackwell Science, Oxford, UK, p.221-434.

Enrich-Prast, A.; Bozelli, R. L.; Esteves, F. A.; Meirelles, F. P. 2004. Lagoas costeiras da Restinga de Jurubatiba: Descrição de suas variáveis limnológicas. In: Rocha, F. D. da R.; Esteves, F. da A. \& Scarano, F. R. (Orgs). Pesquisas de longa duração na Restinga de Jurubatiba: Ecologia, história natural e conservação. Rima, São Carlos, Brasil, p. 245-254.

Esteves, F. A. 1998. Fundamentos de Limnologia. $2^{\text {a }}$ ed. Interciência, Rio de Janeiro, Brasil, 602pp.

Hargreaves, J. A. 1998. Nitrogen biogeochemistry of aquaculture ponds. Aquaculture, 166: 181-212.

Keppeler, E. C. 2005. Características limnológicas da água, sedimento e efluentes em viveiros de crescimento final do camarão-da-amazônia, Macrobrachium amazonicum, submetidos a diferentes níveis de arraçoamento e tipos de despescas. Tese de Doutorado, Universidade Estadual Paulista, Brasil, 89pp.

Keppeler, E. C. 2008. Transparência da água em viveiros de $M a-$ crobrachium amazonicum sob diferentes níveis de arraçoamento e despescas seletivas. Biotemas, 21 (4): 55-64.

Keppeler, E. C.; Valenti, W. C. 2006. Effects of selective harvest of the amazon river prawn, Macrobrachium amazonicum, on pond water, sediment andeffluent. Acta Limnologica Brasiliensia, 18 (2): 110-119.

Kubitza, F. 2003. Qualidade da água no cultivo de peixes e camarões. $3^{\text {a }}$ ed. ESALQ/USP, Jundiaí, Brasil, 229pp.

Macedo, C. F. 2004. Qualidade da água em viveiros de criação de peixes com sistema de fluxo contínuo. Tese de Doutorado, Universidade Estadual Paulista, Brasil, 136pp.

Margalef, R. 1983. Limnología. Omega S.A., Barcelona, España, 1010pp.
Malassen, M.; Valenti, W. C. 2006. Effect of nitrite on larval development of giant river prawn Macrobrachium rosenbergii. Aquaculture, 262: 1292-1298.

Midlen, A.; Redding, T. 2000. Environmental management for aquaculture. Kluwer Academic Publishers, Dordrecht, Netherlands, 223pp.

New, M. B. 2002. Farming freshwater prawns: A manual for the culture of the giant river prawn (Macrobrachium rosenbergii). FAO Fisheries Technical Paper, Rome, Italy, 212pp.

Ono, E. A.; Kubitza, F. 2003. Cultivo de peixes em tanques-rede. USP/ESALQ, Jundiaí, Brasil, 112pp.

Shishehchian, F.; Yusoff, F. M.; Omar, H.; Kamarudin, M. S. 1999. Nitrogenous excretion of Penaeus monodon postlarvae fed with different diets. Marine Pollution Bulletin, 39 (1-12): 224-227.

Schmidt-Nielsen, K. 2002. Fisiologia animal: Adaptação e meio ambiente. Santos Livraria Editora, São Paulo, Brasil, 601pp.

Solorzano, L. 1969. Determination of ammonia in natural waters by the phenolhypochlorite method. Limnology and Oceanography, 14: $799-801$

Preto, B. L.; Pizzato, G. M.; Valenti, W. C. 2008. Uso de bandejas de alimentação na fase de engorda do camarão-da-amazônia, $M a-$ crobrachium amazonicum (Heller, 1862). Boletim do Instituto de Pesca, 34 (1): 125-131.

Statsoft. 1996. Statistica. Version 6.0. Statsoft Company.

Strickland, J. D.; Parsons, T. R. 1960. A manual of seawater analysis. Bulletin of the Fisheries Research Board of Canada, 125: 1-185.

Troppmair, H. 1975. Regiões ecológicas do Estado de São Paulo. Instituto de Geografia da USP, São Paulo, Brasil, 24pp.

Valderrama, J. C. 1981. The simultaneous analysis of total nitrogen and total phosphorus in natural waters. Marine Chemistry, 10: 109-122.

Valenti, W. C. 1989. Efeitos da densidade populacional sobre o cultivo do camarão Macrobrachium rosenbergii (De Man, 1979) no Norte do Estado de São Paulo: Análise quantitativa (Crustacea, Palaemonidae). Tese de Doutorado, Universidade de São Paulo, Brasil, 132pp.

Wetzel, R. G. 1981. Limnologia. Ômega S. A., Barcelona, España, 679pp.

Wetzel, R. G.; Likens, G. E. 1991. Limnological analysis. Springer-Verlag, New York, USA, 391pp.

Zar, J. H. 1984. Biostatistical analysis. $2^{\text {nd }}$ ed. Prentice Hall, Englewood Cliffs, New Jersey, USA, 718pp.

Zimmermann, S. 1998. Manejo da qualidade de água e do sedimento dos viveiros. In: Valenti, W. C. (Ed.). Carcinicultura de água doce: Tecnologia para produção de camarões. FAPESP/IBAMA, Brasília, Brasil, p.165-177. 